\title{
Effects of Vitamin D Supplementation on Depression Status, Selected Pro- inflammatory Biomarkers and Neurotransmitters in Depressive Patients: A Study Protocol
}

\author{
Mina Kaviani ${ }^{1}$, Bahareh Nikooyeh ${ }^{2}$, Hamid Zand $^{2}$, Parichehreh Yaghmaei ${ }^{1}$, Tirang R. Neyestani ${ }^{2 *}$ \\ 1- Department of Biology, Science and Research Branch, Islamic Azad University, Tehran, Iran \\ 2- Faculty of Nutrition and Food Technology, National Nutrition and Food Technology Research Institute, Shahid Beheshti University of Medical \\ Sciences, Tehran, Iran
}

\section{A B S T R A C T}

Background and Objectives: Up to date, several pathophysiological mechanisms are suggested for evolution of depression, including inflammation, neurotransmitter and vitamin D pathways. The aim of this study is to evaluate the effects of vitamin D supplementation on serum 25-hydroxycalciferol [25(OH) D], intact parathyroid hormone (iPTH), some pro-inflammatory biomarkers and neurotransmitters supposedly involved in depression. Furthermore, effects of the vitamin $\mathrm{D}$ are studied on depression status in affected patients.

Materials and Methods: Patients with mild to moderate depression, aged 18-60 y, are participated in the study and randomly assigned into intervention (50,000 IU of cholecalciferol per two weeks) or control (placebo) groups. Duration of the intervention is eight weeks. Demographic and anthropometric parameters, blood pressure, biochemical values and depression status are recorded before and after intervention. Biochemical tests include serum $25(\mathrm{OH}) \mathrm{D}$, iPTH, highsensitivity C-reactive protein (hs-CRP), interleukin-1 $\beta$ (IL-1 $\beta$ ), interleukin-6 (IL-6) and also neurotransmitters involved in depression include platelet serotonin and serum oxytocin.

Conclusions: Several parameters are linked to vitamin D and depression status. Findings of this study can help clarify roles of these parameters, which may further be used in depression preventive and therapeutic strategies.

Keywords: Depression, Vitamin D, RCT, Study protocol

\section{Introduction}

Depression, as a leading cause of individual psychological and physical disabilities and economic problems, has raised the attention of health professionals worldwide $(1,2)$. The global prevalence of depression has been estimated as 3-17\% (WHO data) (2). In Iran, prevalence of the major depressive disorder (MDD), as one of the five diseases with the highest burden on health care system in 2010 (3), is estimated as $4.1 \%$ (4). Depression is described as a multifactorial disorder, which frequently reflects the interplay between psychosocial, genetic, epigenetic, physiologic, neuroendocrine and neuroimmunological factors as well as diet and lifestyle $(1,5,6)$. However, pathogenesis mechanisms and components of depression, including inflammation, neurotransmitters and vitamin $\mathrm{D}$, have already been addressed $(7,8)$. Nevertheless, the true magnitude of these mechanisms and their interactions are not clearly known (5). Increases in pro-inflammatory biomarkers such as high-sensitivity C-reactive protein (hs-CRP), interleukin-1 $\beta$ (IL-1 $\beta$ ) and interleukin-6 (IL-6) prior to onset of depression have raised great attentions (5, 9, 10). However, most of these findings are based on studies that assessed one inflammatory factor (11); hence, further studies are necessary to investigate these factors simultaneously. Furthermore, results from the studies on interactions of pro-inflammatory molecules with other factors in depression (e.g. vitamin D) are contradictory (12). Several studies have reported that insufficient levels of serotonin, as a neurotransmitter involved in mood and behavior, may result in depression (13-15). However, most of these reports are based on serum serotonin measurements in depressive patients (16-18), while platelet serotonin 
measurement is a further accurate alternative of brain serotonin $(15,19)$. It is broadly accepted that dysfunction of oxytocin, another neurotransmitter, includes important roles in mood, behavior, brain development and sociality (21-24). However, verification of oxytocin roles in depression needs further studies. Relatively, vitamin D may include roles in depression $(8,25)$. Hypovitaminosis D has repeatedly been reported in patients with depression from various countries, including Iran (11, 25-27). Nevertheless, information on possible effects of vitamin D supplementation on depression are contradictory (28-31). Examples of vitamin D roles in nervous system include its roles in inflammation reduction, neurotransmitter synthesis and function and brain function and mood $(11,16,32,33)$. However, it is still unclear whether vitamin D affects depression via affecting pro-inflammatory cytokines or associated neurotransmitters. To the best of the authors' knowledge, the current comprehensive study will, for the first time evaluate the effects of vitamin D supplementation on various mechanistic aspects of the depression.

Objectives: The aim of this study is to evaluate the effects of vitamin D supplementation on serum $25(\mathrm{OH})$ D, iPTH, selected pro-inflammatory biomarkers and neurotransmitters involved in depression and on depression status in the depressed patients.

Hypothesis: It was hypothesized that increases in blood vitamin D through administration of 50,000 IU vitamin $\mathrm{D}$ biweekly in patients with mild to moderate depression will result in decreases in selected proinflammatory biomarkers and increases neurotransmitters involved in depression and hence in improvement of depressive status.

\section{Materials and Methods}

Study design. A double-blind, randomized clinical trial (RCT) is designed and carried out for patients with mild to moderate depression and no other psychiatric diseases, who are referred to Outpatient Clinics of Baharloo Hospital, Tehran, Iran, May 2018 to June 2019. Participants are recruited through notifications in the hospital and public places around the hospital. Volunteer patients are registered to refer to psychiatrists for diagnosis. Eligibility assessment of the patients is carried out using structured clinical diagnostic interviews based on Diagnostic and
Statistical Manual of Mental Disorders- $4^{\text {th }}$ ed. (DSM-IV) criteria and Beck Depression Inventory-II (BDI-II) (34). After describing the study protocol for the patients and signing printed informative consents by them, the eligible patients are participated in the study and randomly assigned into intervention (50,000 IU of cholecalciferol per two weeks) or control (placebo) groups. Vitamin D3 supplements and placebos are supplied by Zahravi Pharmaceutical Company, Iran, and are completely similar in appearance and packaging. Randomization is carried out based on the entrance codes of the participants. Time duration of the intervention includes eight weeks (Figure 1).

Calculation of the sample size: Considering an effect size of 0.75 and a power of $80 \%, 28$ patients are assigned to each group (35).

Inclusion criteria: The inclusion criteria include 1) aging 18-60 y; and 2) having mild to moderate depression with no other psychiatric diseases, according to psychiatrists' assessments.

Exclusion criteria: The exclusion criteria include 1) having a history of heart infarction, angina pectoris, stroke, kidney stone and/or high blood pressure (systolic blood pressure higher than 174 and/or diastolic blood pressure higher than $104 \mathrm{~mm} \mathrm{Hg}$ ) (36); 2) having liver disease and/or hyperparathyroidism; 3) being pregnant and/or lactating; 4) reproductive aged women (under 50 years old), who are not receiving adequate contraception; 5) consuming nutritional supplements with vitamin $\mathrm{D}$ since two months prior to intervention; 6) discontinuing the study; and 7) failing to precisely follow the interventional program. The primary outcome includes a significant increase in serum $25(\mathrm{OH})$ D concentration from baseline to Week 8 of intervention. Vitamin $D$ status is categorized based on the following circulating concentrations of calcidiol as deficiency $(<50 \mathrm{nmol}$ $\left.\mathrm{L}^{-1}\right)$, insufficiency $\left(50-75 \mathrm{nmol} \mathrm{L}^{-1}\right)$ and normal (> 75 nmol $\mathrm{L}^{-1}$ ) (37). Secondary outcomes included significant changes in serum pro-inflammatory biomarkers such as IL- $1 \beta$, IL- 6 and hs-CRP and in neurotransmitters such as serum oxytocin and platelet serotonin. Other secondary outcomes included significant changes in serum iPTH as well as depression status (BDI-II score) from baseline to Week 8 of intervention. 


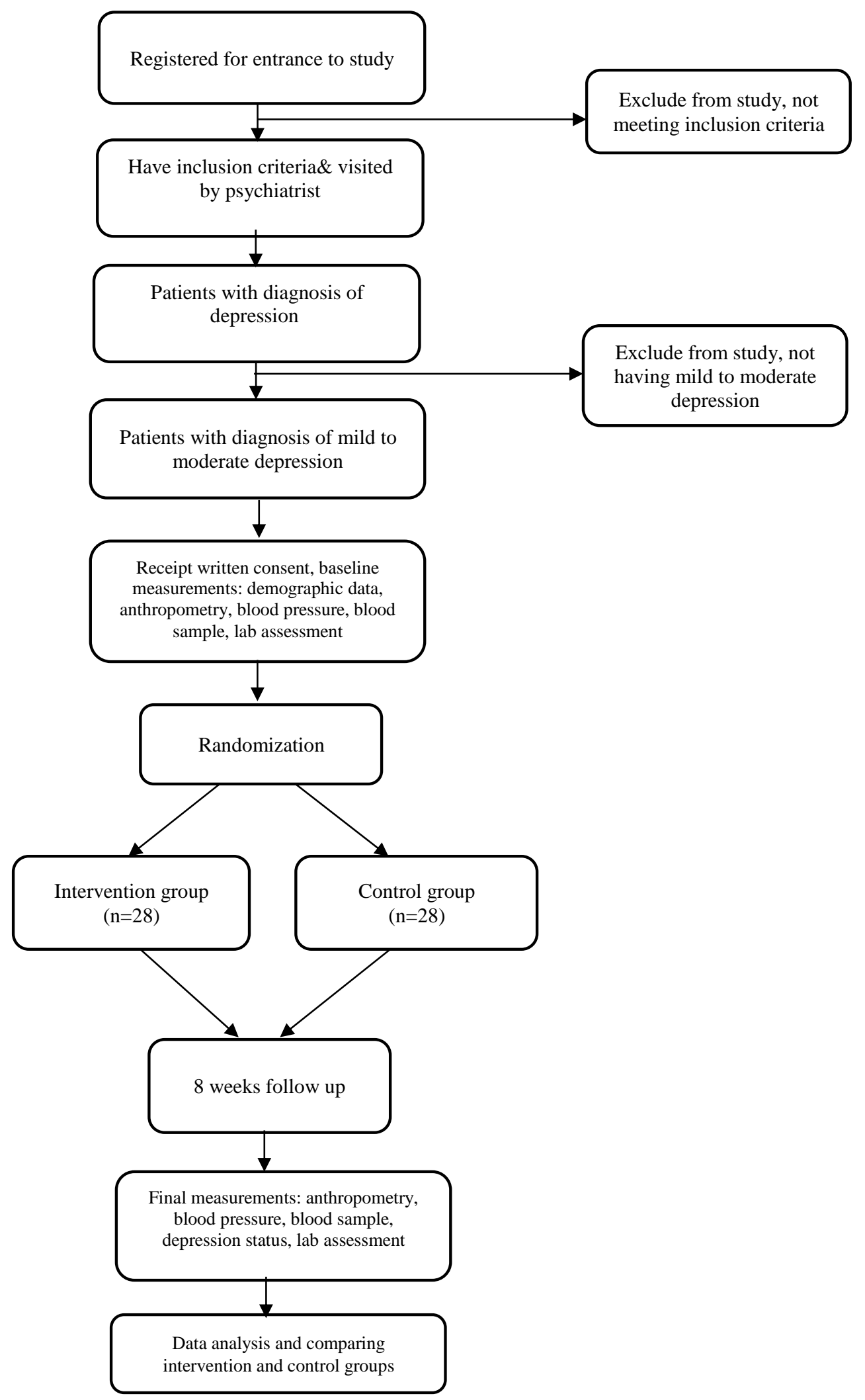

Figure 1. Flow chart of the study design 


\section{Data collection and variables}

Demographic Data: Three study visits are carried out for each participant on Weeks 0,4 and 8. On the first visit, a general socio-demographic questionnaire, including age, sex, educational level, disease history, marital situation, exposure to sunlight (time, duration and body parts), sunscreen use, tobacco and drug uses, alcohol consumption and physical activity, is completed for each participant using face-to-face interview. Then, participants are asked to continue on their usual diet, physical activity and medications during the intervention. In this study, preferably incident cases are enrolled. However, patients with pre-existing depression may also be included. As participants will be randomly allocated to the study groups, it is expected that incident and old cases are similarly distributed within the groups.

Depression status. Depression status of all participants is assessed before and after intervention using BDI-II questionnaire.

\section{Anthropometric measurement and blood pressure:}

Weight is measured to the nearest of $0.1 \mathrm{~kg}$ without shoes and with light clothing using calibrated digital scale (Seca 808; Seca, Hamburg, Germany). Height is measured in standing position (only on the first visit) without shoes to the nearest of $0.1 \mathrm{~cm}$ using standard stadiometer. Body mass index (BMI) $\left[\mathrm{kg}\left(\mathrm{m}^{2}\right)^{-1}\right]$ is calculated by dividing weight $(\mathrm{kg})$ by height $\left(\mathrm{m}^{2}\right)$. Waist circumference is measured to the nearest of 0.1 $\mathrm{cm}$ at the midpoint between the lower rib and iliac crest at the end of expiration and hip circumference is measured to the nearest of $0.1 \mathrm{~cm}$ at the maximum extension of the buttock, horizontal to the floor, using measuring tape (38). Waist to hip ratio (WHR) is calculated by dividing waist circumference to hip circumference. Systolic and diastolic blood pressures are measured in sitting position after 10 min resting using digital sphygmomanometer (BC 08; Beurer, Ulm, Germany).

Blood sampling: A venous blood sample is collected from each participant on the first and third visits (10 $\mathrm{mL} ; 5 \mathrm{~mL}$ in tubes without anticoagulant and $5 \mathrm{~mL}$ in tubes with Ethylenediaminetetraacetic acid (EDTA)) and transferred to Laboratory of Nutrition Research, National Nutrition and Food Technology Research Institute (NNFTRI) using cold box.
Intervention. The participants receive vitamin D supplements or placebos, according to their study groups, on the first and second visits. They are asked to take their pills at the clinic on Weeks 0 and 4 to ensure that at least $50 \%$ of the pills are consumed. In addition to receive a written "instruction of use", participants receive a reminder call for the consumption of remaining pills. Furthermore, the participants are asked to return the pills that are not consumed for any reason on their next visit. Therefore, the adherence assessment is carried out based on the supplement/placebo count and patient self-report.

\section{Laboratory investigation}

Serum separation: Sera are separated from the clotted samples by centrifugation at $800 \mathrm{~g}$ for $20 \mathrm{~min}$ at room temperature (RT). Then, samples are aliquoted in fresh microtubes and stored at $-80{ }^{\circ} \mathrm{C}$ until use.

Platelet separation. Platelets are separated from the whole blood samples by centrifugation at 200 $\mathrm{g}$ for $10 \mathrm{~min}$ at RT to achieve platelet-rich plasma (PRP). Then, the PRP-supernatants are transferred to fresh microtubes and platelets are counted using automatic cell counter (Mythic; Orphee, Switzerland). To collect platelet pellets, an aliquot of PRP with 700,000 platelets $\mu \mathrm{L}^{-1}$ is added to physiological saline up to a total volume of $1 \mathrm{~mL}$ and centrifuged at $4,500 \mathrm{~g}$ for $10 \mathrm{~min}$ at $4{ }^{\circ} \mathrm{C}$. For neutralizing possible effects of vitamin $\mathrm{D}$ on platelet count, the volume of PRP is calculated proportional to the platelet count in the PRP for each sample individually. Then, the supernatant is discarded and $200 \mu \mathrm{L}$ of double-distilled water is added to the pellet and stored at $-80^{\circ} \mathrm{C}$ until use.

Biochemical tests: Biochemical parameters are assessed using enzyme-immunoassay (EIA) method and commercial kits (Table 1).

Ethical issues: Ethical approval for the present study was received from the Ethical Committee of NNFTRI (IR.SBMU.NNFTRI.REC.1396.185). The clinical trial registration code was received from the Iranian Registry of Clinical Trials (IRCTID: IRCT20170926036425N1) and ClinicalTrials.gov (NCT03766074). 
Table 1. Technical information of the commercial kits

\begin{tabular}{lllll}
\hline Parameter & Commercial kit & $\begin{array}{l}\text { Intra-assay } \\
\text { variations }\end{array}$ & $\begin{array}{l}\text { Inter-assay } \\
\text { variations }\end{array}$ & LOD $^{1,2}$ \\
\hline Serum 25(OH)D & Euroimmun EIA kit (Lubeck, Germany) & $3.2-6.9 \%$ & $7-8.6 \%$ & $4 \mathrm{nmol} / \mathrm{L}$ \\
Serum iPTH & Euroimmun EIA kit (Lubeck, Germany) & $2.2-9.5 \%$ & $9.5-11 \%$ & $1.5 \mathrm{pg} / \mathrm{mL}$ \\
Serum IL-1 & Diaclone EIA kit (Besancon, France) & $4.5 \%$ & $8.7 \%$ & $6.5 \mathrm{pg} / \mathrm{mL}$ \\
Serum IL-6 & IBL EIA kit (Hamburg, Germany) & $3.4 \%$ & $5.2 \%$ & $0.92 \mathrm{pg} / \mathrm{mL}$ \\
Serum hs-CRP & Zellbio EIA kit (Ulm, Germany) & $<10 \%$ & $<12 \%$ & $0.01 \mathrm{mg} / \mathrm{L}$ \\
Serum oxytocin & Zellbio EIA kit (Ulm, Germany) & $<10 \%$ & $<12 \%$ & $1 \mathrm{ng} / \mathrm{L}$ \\
Platelet serotonin & Zellbio EIA kit (Ulm, Germany) & $<10 \%$ & $<12 \%$ & $1.2 \mathrm{ng} / \mathrm{mL}$ \\
\hline
\end{tabular}

${ }^{1}$ Based on the manufacturer instruction; ${ }^{2}$ limit of detection

Safety considerations: Based on the upper tolerable level intake of vitamin D for adults (4,000 IU day $\left.{ }^{-1}\right)$ (39), the harmful dose of vitamin D (> 10,000 IU d $\left.{ }^{-1}\right)$ (40) and results from previous studies with higher doses of vitamin D $\left(50,000 \mathrm{IU} \mathrm{w}^{-1}\right)(12)$, the used dose of vitamin D in this study $(50,000$ IU per two weeks) seems safe.

\section{Statistical analyses}

Data are expressed as mean \pm SD (standard deviation) to describe quantitative data and absolute or relative frequencies of the qualitative data. Shapiro-Wilks test is used for evaluating the normality of data distribution. Chi-square test is used to compare qualitative variables between the groups at baseline. Based on the study design, two groups are investigated within two time periods (before and after intervention); therefore, paired-sample $t$-test or Wilcoxon test (based on the normality distribution of data) is used to compare within-group changes. To compare between-group changes, independent sample $t$-test or Mann-Whitney test (based on the normality distribution of data) is used. Evaluation of correlations between the two groups of data is carried out using Pearson (for data with normal distribution, r) or Spearman (for non-normal distribution, $r_{\mathrm{s}}$ ) test. Single or multiple regression test is used to assess relationships between the changes in indicators during the study. The significance level is set at $P<0.05$. Data are analyzed using Statistical Package for Social Sciences (SPSS) Software v.21 (SPSS Inc., Chicago, IL, USA).

\section{Discussion}

Depression is well-known as a multifactorial mood disorder (1). Although several parameters such as inflammation, neurotransmitters and vitamin D are suggested to be involved in pathophysiology of depression $(7,8)$, possible associations between the various dimensions of depression must be clarified (5). Results from the previous studies have shown that hypovitaminosis $\mathrm{D}$ is prevalent in patients with depression (11, 25-27); however, results of studies on effects of vitamin D supplementation on depression are contradictory (28-31). Despite suggested roles for vitamin $\mathrm{D}$ in inflammation, neurotransmitter metabolism and mood status (11, 16, 32, 33), associations of vitamin D with depression status need more elucidation. In conclusion, results of this study can help clarify pathophysiological mechanisms involved in depression. Furthermore, these results can be used in future approaches of the depression prevention as well as depression treatment.

\section{Strengths of the study}

Strengths of the study include 1) designing a doubleblind randomized clinical study; 2) assessing three mechanisms involved in depression simultaneously; 3) evaluating correlations between oxytocin and vitamin $\mathrm{D}$ in patients with depression for the first time; 4) assessing platelet serotonin concentration as an accurate alternative of brain serotonin concentration; and 5) investigating confounding factors (e.g. sun exposure, physical activity).

\section{Financial disclosure}

The authors declare no financial interest.

\section{References}

1. Gururajan A, Clarke G, Dinan TG, Cryan JF. Molecular biomarkers of depression. Neurosci Biobehav Rev. 2016;64:101-33.

2. Marcus M, Yasamy MT, Van Ommeren M, Chisholm D, Saxena S. Depression: A global public health concern2012. 6-8 p.

3. INCD: National Action Plan for Prevention and Control of Non-Communicable Diseases and the Related Risk Factors in the Islamic Republic of Iran, 2015-2025. 
Iran: Iranian national committee for NCDs prevention and control; 2015 september, 2015.

4. Sadeghirad B, Haghdoost AA, Amin-Esmaeili M, Ananloo ES, Ghaeli P, Rahimi-Movaghar A, et al. Epidemiology of major depressive disorder in iran: a systematic review and meta-analysis. Int $\mathrm{J}$ Prev Med. 2010;1(2):81-91.

5. Berk M, Williams LJ, Jacka FN, O'Neil A, Pasco JA, Moylan S, et al. So depression is an inflammatory disease, but where does the inflammation come from? BMC Med. 2013;11:200.

6. Shabbir F, Patel A, Mattison C, Bose S, Krishnamohan $\mathrm{R}$, Sweeney E, et al. Effect of diet on serotonergic neurotransmission in depression. Neurochem Int. 2013;62(3):324-9.

7. Moylan S, Maes M, Wray NR, Berk M. The neuroprogressive nature of major depressive disorder: pathways to disease evolution and resistance, and therapeutic implications. Mol Psychiatry. 2013;18(5):595-606.

8. Verduijn J, Milaneschi Y, Schoevers RA, van Hemert AM, Beekman AT, Penninx BW. Pathophysiology of major depressive disorder: mechanisms involved in etiology are not associated with clinical progression. Transl Psychiatry. 2015;5:e649.

9. Lichtblau N, Schmidt FM, Schumann R, Kirkby KC, Himmerich H. Cytokines as biomarkers in depressive disorder: current standing and prospects. Int Rev Psychiatry. 2013;25(5):592-603.

10. Miller AH, Haroon E, Raison CL, Felger JC. Cytokine targets in the brain: impact on neurotransmitters and neurocircuits. Depress Anxiety. 2013;30(4):297-306.

11. Shin YC, Jung CH, Kim HJ, Kim EJ, Lim SW. The associations among vitamin D deficiency, C-reactive protein, and depressive symptoms. J Psychosom Res. 2016;90:98-104.

12. Sepehrmanesh Z, Kolahdooz F, Abedi F, Mazroii N, Assarian A, Asemi Z, et al. Vitamin D Supplementation Affects the Beck Depression Inventory, Insulin Resistance, and Biomarkers of Oxidative Stress in Patients with Major Depressive Disorder: A Randomized, Controlled Clinical Trial. J Nutr. 2016;146(2):243-8.

13. Mottolese R, Redoute J, Costes N, Le Bars D, Sirigu A. Switching brain serotonin with oxytocin. Proc Natl Acad Sci U S A. 2014;111(23):8637-42.

14. Homan P, Neumeister A, Nugent AC, Charney DS, Drevets WC, Hasler G. Serotonin versus catecholamine deficiency: behavioral and neural effects of experimental depletion in remitted depression. Transl Psychiatry. 2015;5:e532.

15. Raz L, Hunter LV, Dowling NM, Wharton W, Gleason CE, Jayachandran $M$, et al. Differential effects of hormone therapy on serotonin, vascular function and mood in the KEEPS. Climacteric. 2016;19(1):49-59
16. Patrick RP, Ames BN. Vitamin D and the omega-3 fatty acids control serotonin synthesis and action, part 2: relevance for ADHD, bipolar disorder, schizophrenia, and impulsive behavior. Faseb j. 2015;29(6):2207-22.

17. Muss C, Mosgoeller W, Endler T. Mood improving Potential of a Vitamin Trace Element Composition--A randomized, double blind, placebo controlled clinical study with healthy volunteers. Neuro Endocrinol Lett. 2016;37(1):18-28.

18. Kaneko I, Sabir MS, Dussik CM, Whitfield GK, Karrys A, Hsieh JC, et al. 1,25-Dihydroxyvitamin D regulates expression of the tryptophan hydroxylase 2 and leptin genes: implication for behavioral influences of vitamin D. Faseb j. 2015;29(9):4023-35.

19. Peitl V, Vidrih B, Karlovic Z, Getaldic B, Peitl M, Karlovic D. Platelet serotonin concentration and depressive symptoms in patients with schizophrenia. Psychiatry Res. 2016;239:105-10.

20. Audhya T, Adams JB, Johansen L. Correlation of serotonin levels in CSF, platelets, plasma, and urine. Biochim Biophys Acta. 2012;1820(10):1496-501.

21. Afinogenova Y, Schmelkin C, Plessow F, Thomas JJ, Pulumo R, Micali N, et al. Low Fasting Oxytocin Levels Are Associated With Psychopathology in Anorexia Nervosa in Partial Recovery. J Clin Psychiatry. 2016;77(11):e1483-e90.

22. Sanchez-Vidana DI, Chan NM, Chan AH, Hui KK, Lee $\mathrm{S}$, Chan HY, et al. Repeated treatment with oxytocin promotes hippocampal cell proliferation, dendritic maturation and affects socio-emotional behavior. Neuroscience. 2016;333:65-77.

23. Domes G, Normann C, Heinrichs M. The effect of oxytocin on attention to angry and happy faces in chronic depression. BMC Psychiatry. 2016;16:92.

24. Jobst A, Sabass L, Hall D, Brucklmeier B, Buchheim A, Hall J, et al. Oxytocin plasma levels predict the outcome of psychotherapy: A pilot study in chronic depression. J Affect Disord. 2018;227:206-13.

25. Lang UE, Beglinger C, Schweinfurth N, Walter M, Borgwardt S. Nutritional aspects of depression. Cell Physiol Biochem. 2015;37(3):1029-43.

26. Kerr DC, Zava DT, Piper WT, Saturn SR, Frei B, Gombart AF. Associations between vitamin D levels and depressive symptoms in healthy young adult women. Psychiatry Res. 2015;227(1):46-51.

27. Nabizade-Asl L, Mozaffari-Khosravi H, YassiniArdekani S, Hadi Nodoushan H, Fallahzadeh $H$. Vitamin D Status in Patients with Depression Referred to Specialized Clinics Affiliated to Shahid Sadoughi University of Medical Sciences, Yazd-2011. Tolooebehdasht. 2012;11(1):9-19.

28. Nerhus M, Berg AO, Kvitland LR, Dieset I, Hope S, Dahl SR, et al. Low vitamin D is associated with negative and depressive symptoms in psychotic disorders. Schizophr Res. 2016;178(1-3):44-9. 
29. Li G, Mbuagbaw L, Samaan Z, Falavigna M, Zhang S, Adachi JD, et al. Efficacy of vitamin D supplementation in depression in adults: a systematic review. J Clin Endocrinol Metab. 2014;99(3):757-67.

30. Anglin RE, Samaan Z, Walter SD, McDonald SD. Vitamin D deficiency and depression in adults: systematic review and meta-analysis. Br J Psychiatry. 2013;202:100-7.

31. Gowda U, Mutowo MP, Smith BJ, Wluka AE, Renzaho AM. Vitamin D supplementation to reduce depression in adults: meta-analysis of randomized controlled trials. Nutrition. 2015;31(3):421-9.

32. Libuda L, Antel J, Hebebrand J, Focker M. [Nutrition and mental diseases : Focus depressive disorders]. Nervenarzt. 2017;88(1):87-101.

33. Caldwell JD, Londe K, Ochs SD, Hajdu Z, Rodewald A, Gebhart VM, et al. Three steroid-binding globulins, their localization in the brain and nose, and what they might be doing there. Steroids. 2019;142:48-54.

34. Wang YP, Gorenstein C. Assessment of depression in medical patients: a systematic review of the utility of the Beck Depression Inventory-II. Clinics (Sao Paulo). 2013;68(9):1274-87.

35. Wang Y, Liu XJ, Robitaille L, Eintracht S, MacNamara E, Hoffer LJ. Effects of vitamin $\mathrm{C}$ and vitamin $\mathrm{D}$ administration on mood and distress in acutely hospitalized patients. Am J Clin Nutr. 2013;98(3):70511.

36. Kjaergaard $M$, Waterloo $\mathrm{K}$, Wang CE, Almas B, Figenschau Y, Hutchinson MS, et al. Effect of vitamin $\mathrm{D}$ supplement on depression scores in people with low levels of serum 25-hydroxyvitamin D: nested casecontrol study and randomised clinical trial. $\mathrm{Br} \mathrm{J}$ Psychiatry. 2012;201(5):360-8.

37. Rosen CJ, Abrams SA, Aloia JF, Brannon PM, Clinton SK, Durazo-Arvizu RA, et al. IOM committee members respond to Endocrine Society vitamin D guideline. J Clin Endocrinol Metab. 2012;97(4):1146-52.

38. Sebo P, Haller D, Pechere-Bertschi A, Bovier P, Herrmann F. Accuracy of doctors' anthropometric measurements in general practice. Swiss Med Wkly. 2015;145:w14115.

39. (NIH) NIoH. Vitamin D, fact sheet for consumers. 2016.

40. Holick MF, Binkley NC, Bischoff-Ferrari HA, Gordon CM, Hanley DA, Heaney RP, et al. Evaluation, treatment, and prevention of vitamin D deficiency: an Endocrine Society clinical practice guideline. J Clin Endocrinol Metab. 2011;96(7):1911-30. 